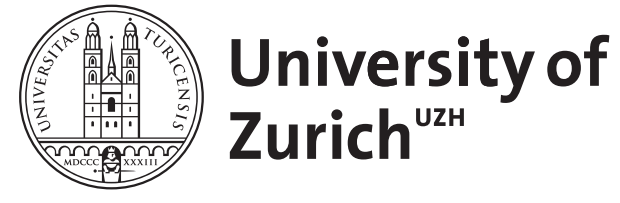

\title{
Wielands Literaturbegriff: transformierte Tradition
}

\author{
Zeuch, Ulrike
}

DOI: https://doi.org/10.1515/9783110240375.1.37

Posted at the Zurich Open Repository and Archive, University of Zurich ZORA URL: https://doi.org/10.5167/uzh-95037

Book Section

Published Version

Originally published at:

Zeuch, Ulrike (2010). Wielands Literaturbegriff: transformierte Tradition. In: Erhart, Walter; van Laak, Lothar. Wissen - Erzählen - Tradition : Wielands Spätwerk. Berlin / New York: Walter de Gruyter GmbH, 37-52.

DOI: https://doi.org/10.1515/9783110240375.1.37 


\section{Wielands Literaturbegriff: transformierte Tradition}

Die Ambivalenz in der Legitimierung der Literatur als wahr durch Wirklichkeitstreue oder durch Fiktionalität als eine andere Art der Wahrheit ist ein Erbe der wirkungsmächtigen, aber dissonanten Auseinandersetzung mit Horaz' Wahrscheinlichkeitspostulat als Kriterium für Wahrheit seit der Frühen Neuzeit. Beide Legitimierungen zielen dabei auf den Anspruch ab, dass Literatur, indem sie etwas Wahres darstellt, zugleich etwas Allgemeines repräsentiert.

Im Votum für Fiktionalität als eine andere Art der Wahrheit geht die seit Scaliger verbindliche Horaz-Nachfolge in die Rebellion; das Votum für Fiktionalität als solche stellt aber keine Lösung des Problems dar. Denn es gibt keine einhellige Antwort auf die Frage, was wirklich und was objektiv ist. Auch stellt das Votum für den Wahrheitsanspruch der Fiktionalität keine plausible Alternative dar. Denn fiktionale Wahrheit ist entweder etwas subjektiv Vorgestelltes oder konsensuell als wahr Beglaubigtes, nicht aber objektiv wahr. ${ }^{1}$ Dass es noch eine dritte Möglichkeit gibt, Wahrheit der Literatur zu begründen, ist durch die Poetik des Aristoteles implizit zwar präsent, spielt aber seit Scaliger explizit keine Rolle. ${ }^{2}$

\section{Welche Position nimmt Wieland innerhalb dieses Problemfeldes ein?}

Dass Wielands Literaturbegriff in Bezug auf die Wahrheitsbegründung Horaz' Ars poetica verpflichtet ist, scheint keiner weiteren Bestätigung zu bedürfen; es gilt als communis opinio der Forschung. ${ }^{3} \mathrm{Ob}$ aber auch die seit der Frühen Neuzeit nicht minder wirkungsmächtige Poetik des Aristoteles

1 Vgl. Kendall L. Walton: „Furcht vor Fiktionen«. In: Fiktion, Wahrheit, Wirklichkeit. Philosophische Grundlagen der Literaturtheorie. Hg. v. Maria E. Reicher. Paderborn 2007, S. 94-II9, hier S. Ioo.

2 Vgl. Ulrike Zeuch: "Das Allgemeine als Gegenstand der Literatur. Scaligers Begriff des Allgemeinen und seine stoischen Prämissen". In: Poetica 34 (2002) H. I-2, S. 99-I24.

3 Klaus Manger: »Aristipp in Wielands Horaz-Übersetzungen von 1782 und 1786 «. In: Christoph Martin Wieland: Aristipp und einige seiner Zeitgenossen. Hg. v. Klaus Manger. Frankfurt/M. 1988 (Bibliothek deutscher Klassiker 28; hier im Text zitiert mit der Sigle SW), S. I04I-I050; Manfred Fuhrmann: "Wieland und Horaz«. In: Christoph Martin Wieland: Übersetzung des Horaz. Hg. v. Manfred Fuhrmann. Frankfurt/M. 1986 (Bibliothek deutscher Klassiker Io; hier im Text zitiert mit der Sigle ÜH), S. Io82-I089. 
bei Wieland nachwirkt, ist in der Forschung bislang nicht thematisiert, was auch nachvollziehbar ist angesichts der durch Wielands Euvre selbst nahegelegten Dominanz des Horaz.

In meinem Beitrag werde ich dieser Frage nachgehen und dafür (I) die Gemeinsamkeiten und Unterschiede von Aristoteles' und Horaz' Gegenstandsbestimmung der Literatur benennen, (2) die Probleme anführen, die sich im Laufe der Rezeption der beiden antiken Dichtungstheorien seit der frühen Neuzeit für das I8. Jahrhundert und damit für Wieland ergeben, und schließlich (3) Wielands Position innerhalb dieses wirkungsgeschichtlichen Feldes bestimmen. Dadurch wird sich - das sei an dieser Stelle bereits vorweggenommen - das Bild von Wielands Affinität zu Horaz' Poetik modifizieren.

\section{Was soll Literatur Wieland zufolge leisten?}

Wieland lässt Aristipp im gleichnamigen Roman Aristipp und einige seiner Zeitgenossen, dem letzten großen Roman Wielands (veröffentlicht 1798 bis I802), in einem Brief Aristipps an Lais von einem Gemälde des Malers Parrhasius berichten. Aristipp nennt erst die Aufgabe, die sich Parrhasius gestellt hat: das Volk Athens zu schildern, ${ }^{4}$ und zwar "von der schönen und hässlichen Seite, mit allen ihren Tugenden und Lastern, Ungleichheiten, Launen und Widersprüchen mit sich selbst, zugleich und auf einen Blick« (AZ, S. I67). Dann erläutert Aristipp, wie dieser vorgegangen ist, um das Ziel: »die Veränderlichkeit und Vielgestaltigkeit des alle möglichen Widersprüche in sich vereinigenden Charakters des Athenischen Demos allegorisch darzustellen " (AZ, S. I69), zu erreichen: Parthasius hat tableauartig eine Volksversammlung in Athen dargestellt, mit über hundert Menschen, die, um drei Personen zentriert, in Gruppen geteilt sind.

Dargestellt ist der Moment, da die Volksversammlung im Begriff ist, sich aufzulösen. Vorangegangen sind drei Handlungen: Der Beschluss einer militärischen Aktion, die Billigung des Staatshaushalts und ein Todesurteil. Die erste Hauptperson ist der Demagoge, eitel, selbstgewiss, machtlüstern und manipulativ. Die um den Demagogen Gescharten spiegeln dessen Eitelkeit und Selbstgewissheit (AZ, S. I67); aber nicht nur: Es gibt auch die Zweifler, Melancholiker, Skeptiker. Die zweite Hauptperson ist der Schatzmeister Athens, erleichtert, dass das Volk seine Bilanzen akzeptiert hat; gleichwohl zeigt er per Profession ein sorgenvolles Gesicht.

4 AZ, S. I66; zum Verfahren des Schilderns als eines zentralen Terminus' im I8. Jahrhundert für den zumindest intendierten Anspruch, authentisch wiederzugeben, was ist, im Unterschied zur Beschreibung als immer schon Bedeutung zuschreibender Interpretation, vgl. Clifford Geertz: Dichte Beschreibung. Beiträge zum Verstehen kultureller Systeme. Frankfurt/M. ${ }^{5}$ I997, S. $7 \mathrm{ff}$. 
Die um ihn Gescharten sind Schmeichler, Schmarotzer und genusssüchtig (AZ, S. I68); aber auch da gibt es Ausnahmen: diejenigen, die die Rechnungen des Finanzministers nachrechnen und zu einem anderen Ergebnis kommen, das eher den Staatsbankrott ankündigt. Die dritte Hauptperson ist der zum Tode Verurteilte, ein Feldherr; dieser selbst ist gefasst, entschlossen und innerlich gestärkt; die ihn begleiten, zeigen in unterschiedlichen Graden Gefühle der Trauer, des Mitleids, der Anteilnahme. Aber es gibt auch die Schadenfreudigen, die Denunzianten, die Meineidigen und schließlich einen, der im Nachhinein bereut, für die Verurteilung gestimmt zu haben.

Abgesehen von den drei Hauptgruppen gibt es weitere, die an dem Geschehen keinen Anteil nehmen, sondern ihren privaten Vergnügungen nachgehen.

Dargestellt werden, so Aristipp, Gefühle als Voraussetzungen von Handlungen, sowie Gefühle als Reaktionen auf Handlungen von moralischer Relevanz; diese Darstellung soll Einsicht der "wirklichen Menschen" (AZ, S. I70) erlauben, der Menschen, "wie sie leiben und leben, und des Laufs der Welt, nicht wie wir ihn gern hätten, sondern wie er ist " (AZ, S. I7of.).

Nachdem Aristipp das Bild beschrieben, d.h. eigentlich: in eine durch Sprache vermittelte Bedeutung übersetzt hat, beurteilt er das Ergebnis: Dargestellt ist nicht die personifizierte Idee des Volkes im Sinne eines Allgemeinen, sondern »eine Menge einzelner Glieder dieses Volks" in einem Moment (AZ, S. 169). Zu einer einheitlichen Idee eines allgemeinen Volkscharakters werden diese Einzelheiten nur im betrachtenden Rezipienten, aber selbst dann gilt, dass sie nur die eines Volkes »in einer jeden Demokratie« ist, weder aber jedes Staatssystems (AZ, S. I69) noch, müsste man ergänzen, zu allen Zeiten.

Das Problem, welches hier verhandelt wird, ist folgendes: Wie lässt sich etwas Mannigfaltiges so darstellen, dass die Vielheit weder in Disparates zerfällt noch sich in der Verschiedenes einheitlich fassenden Allgemeinheit verliert? In der ersten Fassung der Geschichte des Agathon ${ }^{5}$ von I766/67 nennt Wieland ein Beispiel, da einzelne Momente zu einem Gesamteindruck zusammenstimmen; in einem solchen Fall stimmt alles, d.h. stimmen alle einzelnen Momente "mit dem Begriff überein" (GA, S. 246). Agathon findet sich im Haus seines Vater und wird von diesem in einen Saal geführt, dessen Mauern vielleicht nicht zufällig von »einem der besten Schüler des Parrhasius" bemalt worden sind. "Die Tafel, das Gerät, die Aufwärter, alles stimmte mit dem Begriff überein, den ich mir bereits von dem Geschmack und dem Stande des Haus-Herrn gemacht hatte" (GA, S. 246).

5 Christoph Martin Wieland: Geschichte des Agathon. Hg. v. Klaus Manger. Frankfurt/M. 1986 (Bibliothek deutscher Klassiker II, hier im Text zitiert mit der Sigle GA) 
Im Aristipp ist die Messlatte für Kunst nicht nur höher gesteckt, auch das Darzustellende ist ungleich komplexer: Nicht nur der Charakter eines einzelnen Menschen, sondern eines ganzen Volks soll repräsentiert werden. Wieland lässt im weiteren Verlauf des Romans Parrhasius auch selbst zu Wort kommen. Auf die Frage, ob er tatsächlich den »Karakter des Athenischen Volks" (AZ, S, 278) habe darstellen wollen, antwortet dieser, das habe er zwar wollen, aber bald eingesehen, dass das auf nur einer Tafel zu leisten unmöglich sei, weswegen er auf den Einfall gekommen sei, statt allegorischer Personifizierungen von Charaktereigenschaften des athenischen Demos eine Volksversammlung in Auflösung begriffen darzustellen, und »zwar so, daß man aus den verschiedenen Gruppen erraten könnte, was unmittelbar vorher verhandelt und beschlossen worden, was dieser und jener für eine Rolle dabei gespielt habe« (AZ, S. 279).

Statt einer statischen Zustandsbeschreibung zielt Parrhasius also auf die Darstellung der Genese bestimmter Handlungen, ihrer Vorgeschichte sowie ihrer Akteure ab, deren einheitliche Fassung im Begriff dem Rezipienten obliegt - ein nicht nur kluger, sondern insofern auch wirkungsmächtiger Einfall, als dieser Einfall tatsächlich in der Folge zu einer Verlagerung von einer Produktions- zu einer Rezeptionsästhetik geführt hat. ${ }^{6}$ Implizit vorausgesetzt ist dabei, dass die Darstellung der Vorgeschichte, die dem Rezipienten eine Rekonstruktion der zeitlichen Abfolge ermöglichen soll, zugleich Aufschluss erlaube über die Ursachen und Folgen, der causae et effectus, der dargestellten Handlung(en). Die zeitliche Abfolge von Handlungen hat mit der Kausalität jedoch nur am Rande etwas zu tun. Insofern intendiert Parrhasius mit seinem Bild etwas, das er gar nicht leisten kann: die Darstellung von seelischen Handlungen im Äußeren: Gesten, Mimik, Körperbewegungen. Und von dem Rezipienten wird ebenso Unmögliches verlangt: von etwas Äußerem, das so oder anders gedeutet werden kann, auf etwas Inneres zu schließen.

Parrhasius meint, ihm sei es gelungen, "alle die verschiedenen Züge, woraus der Karakter der Athener zusammengesetzt ist, auf die natürlichste Art in Handlung und Kontrast zu setzen" (AZ, S. 279). Aristipp selbst sieht das anders. Und folglich entspricht die Darstellung des athenischen Demos durch Parrhasius nicht Aristipps Erwartung, ist mithin - so sein Urteil - nicht wahr. Es sei aber, so Aristipp abschließend, nicht das Versäumnis des Malers; vielmehr liege es in den Grenzen der Kunst begründet. Niemand könne besser malen als Parrhasius (AZ, S. 170). Leistet eben dies dann aber die Literatur? Und wie kommt es überhaupt zu dem

6 Alexandra Pontzen: Künstler ohne Werk. Modelle negativer Produktionsästhetik in der Künstlerliteratur von Wackenroder bis Heiner Müller. Berlin 2000; Stefanie Hüttinger: Der Tod der Mimesis als Ontologie und ihre Verlagerung zur mimetischen Rezeption. Eine mimetische Rezeptionsästhetik als postmoderner Ariadnefaden. Frankfurt/M. u. a. I994. 
Ansinnen, Kunst oder Literatur sollten etwas Allgemeines und Wahres, wie es ist, zugleich darstellen? In der Geschichte der Literaturtheorie hat es auf die Frage, ob die Literatur Wahres darstelle, im Wesentlichen zwei Antworten gegeben:

\section{Allgemeinbegriff bei Aristoteles und Horaz}

Indem die Literatur etwas Allgemeines im Unterschied zur Geschichtsschreibung darstellt, wie Aristoteles im 9. Kapitel der Poetik ausführt, ist sie wahr. ${ }^{7}$ Das in der Poetik gemeinte Allgemeine in der Tragödie ist, dass ein in seinem charakterlichen Habitus bestimmter Mensch in einer bestimmten Situation auf bestimmte Weise handelt, etwa dass ein zu übermäßigem Zorn Neigender, wenn er sich in seiner Ehre verletzt fühlt, auch übermäßig zornig handelt. Der griechische Terminus ist $k \alpha$ ò̀ $\lambda \circ v$ (Poetik, I45I b8). Es ist ein konkret Allgemeines; ${ }^{8}$ nicht Handlung als solche ist gemeint, sondern eine bestimmte Handlung einer bestimmten Person in einer bestimmten Situation.

Aristoteles zufolge ist die Nachahmung einer Handlung in diesem Sinne Gegenstand der Tragödie (Poetik, I449b36-I450aI), nicht ein Substrat, von dem Verschiedenes prädizierbar ist, etwa eine Person mit Eigenschaften. Auch geht es nicht um ein in der Vorstellung Gegebenes im Sinne historischer Einmaligkeit, dessen Umgebung und Umstände jeweils andere sind. Das ist nach Aristoteles Sache der Geschichtsschreibung (Poetik, I45Ib6-7, I459a2I-24). Zwar hat die Tragödie u.a. eine Handlung zum Gegenstand, wie sie ist oder war. Aber mit dem 'wie sie ist ist keine historisch getreue, authentische Wiedergabe beabsichtigt; denn die Tragödie kann ebenso auch Handlung zum Gegenstand haben, wie sie der Mutmaßung oder aller Wahrscheinlichkeit nach zu sein scheint oder wie sie sein sollte (Poetik, I460b7-II).

Es gibt noch eine zweite, ebenfalls sehr wirkungsmächtige Variante dieses Allgemeinen: Indem die Literatur darstellt, was der Wahrscheinlichkeit entspricht und damit der allgemeinen Erfahrung nahe kommt bzw. ihr nicht widerspricht, genügt sie dem Anspruch auf Wahrheit, wie Horaz zu Beginn der Ars poetica am Beispiel frei imaginierter Lebewesen erläutert. ${ }^{9}$

7 Vgl. dazu Arbogast Schmitt: »Was macht Dichtung zur Dichtung? Zur Interpretation des neunten Kapitels der Aristotelischen Poetik (I45Ia36-biI)«. In: Mimesis - Repräsentation - Imagination. Literaturtheoretische Positionen von Aristoteles bis zum Ende des I8. Jahrhunderts. Hg. v. Jörg Schönert u. Ulrike Zeuch. Berlin, New York 2004, S. 65-95.

8 Vgl. dazu Aristoteles: Poetik, übers. und erl. v. Arbogast Schmitt. Darmstadt 2008.

9 Zur Rezeption dieser beiden Varianten einer Darstellung des Wahren innerhalb der Literatur seit der Frühen Neuzeit bis zum 20. Jahrhundert vgl. Burghard Damerau: Die Wahrheit der Literatur. Glanz und Elend der Konzepte. Würzburg 2003. 
Das Allgemeine als Gegenstand der Literatur wird von Horaz dabei auf dreierlei Weise erörtert. Als allgemein bezeichnet Horaz ein für eine Gruppe von Menschen typisches Merkmal, ob nun für eine nach Alter, Herkunft, sozialer Stellung oder Beruf unterschiedene Gruppe. ${ }^{10}$ Dieses Merkmal oder eine bestimmte Anzahl von bestimmten Merkmalen ist gewonnen aus der Wiederholung gleicher Erfahrung. Es ist folglich ein Allgemeines, das dem Konsens und der üblichen, allgemeinen Realitätserfahrung entspricht und insofern Glaubwürdigkeit genießt. ${ }^{11}$ Damit wird das aus der Empirie abgeleitete konstante Merkmal zugleich in den Status eines Ideals versetzt, was natürlich nur im Bereich des ethisch Vorbildhaften gelten kann, denn der Inbegriff des Schlechten, auch wenn es etwas Allgemeines ist, kann kein Vorbild sein. Es wird zum »exemplar vitae morumque" (Ars poetica, Z. 317).

Die Konstanz bezieht sich jedoch nicht nur auf eine Gruppe von Menschen, bei denen sich dieselbe Beobachtung eines bleibenden und deshalb typischen Merkmals machen lässt, sondern auch bei ein und derselben Person in verschiedenen Situationen: Sie muss immer sie selbst, aus einem Guss und bestimmt sein durch ein signifikantes Merkmal, das sich in allen verschiedenen Zuständen und Handlungen durchhält ${ }^{12}$ und als Merkmal mit dem inneren Affekt übereinstimmender äußerer Ausdruck ist. ${ }^{13}$ Laut Horaz (Ars poetica, Z. I25ff.) ist der Gegenstand der Literatur die darzustellende Person, bestimmt durch eine vorherrschende Leidenschaft, die ihr eigentümlich ist und sie von allen anderen unterscheidet.

Nach Horaz gilt es, die Leidenschaft darzustellen, nicht die Handlung, die unterschiedliche Subjekte der Handlung und unterschiedliche Motive und Ursachen aufweist. Der darzustellende Affekt bestimmt die Person von Anfang bis Ende durchgängig. Die Konstanz des Affekts macht das literarische Werk zu einem einfachen, einheitlichen (»simplex dumtaxat et unum", Ars poetica, Z. 23) und ganzen ("totum", Ars poetica, Z. 34), nicht die Einheit der Handlung, d.h. der sachliche und damit notwendige Zusammenhang einzelner Handlungen.

Und noch in einer dritten Hinsicht wird von Allgemeinem gesprochen. Die Literatur dient der Versinnlichung allgemeiner Handlungsmaximen

10 Quintus Flaccus Horatius: "Ars poetica«. In: Opera. Ed. D. R. Shackleton Bailey, Ed. 4. Monachii u.a. 200I, Z. II4ff., I54ff. und 316.

11 Beispiele gegen die Glaubwürdigkeit, nämlich »vanae species" (Z. 7 f.), Horaz: Ars poetica (Anm. Io), Z. Iff., also z. B. phantastische Zusammensetzungen aus Menschenkopf, Pferdehals und Gliedmaßen diverser anderer Tiere.

12 Horaz: Ars poetica (Anm. IO), Z. I25-I27: »siquid inexpertum scaenae conmittis et audes / personam formare novam, servetur ad imum, / qualis ab incepto processerit, et sibi constet".

13 Horaz: Ars poetica (Anm. Io), Z. Io8-III: »format enim natura prius nos intus ad omnem / fortunarum habitum: iuvat aut inpellit ad iram / aut ad humum maerore gravi deducit et angit: / post effert animi motus interprete lingua». 
oder Sentenzen (Ars poetica, Z. 333-336). Letztere sind allgemein, insofern sie als immer gleiche Richtschnur des Handelns in verschiedenen Situationen dienen sollen.

Für alle drei Varianten des Allgemeinen gilt, dass dieses Allgemeine "simplex dumtaxat et unum" (Ars poetica, Z. 23), einheitlich ist und keinen immanenten Widerspruch aufweist. Von allem, was diese Einheitlichkeit stört, ist abzusehen. Für alle drei Varianten des Allgemeinen gilt ferner, dass etwas Allgemeines individualisiert wird; es geht darum, "proprie communia dicere" (Ars poetica, Z. I28). Zwar gesteht Horaz ein, dass es schwierig sei, "proprie communia dicere". Gleichwohl hält er - trotz dieses Eingeständnisses - daran fest: Literatur personifiziert allgemeinverbindliche Tugenden oder Handlungsmaßstäbe wie etwa die pietas; sie stellt nicht, wie Aristoteles meint, eine bestimmte handelnde Person im Hinblick auf dasjenige dar, was an deren Handlungen weder zufällig noch beliebig ist, sondern bei einem Charakter mit bestimmten Eigenschaften sich in einer bestimmten Situation mit Gewissheit und insofern verallgemeinerungsfähig ergibt.

\section{Probleme der beiden Allgemeinbegriffe}

In der Geschichte der Literaturtheorie sind diese beiden Antworten Anlass zu vielfältigen Deutungen gewesen. Was hat Aristoteles mit 'Allgemeinem gemeint? So man davon ausgeht, es handele sich um Mimesis menschlicher Handlung: Ist dieses Allgemeine ein Vorbild, ein Exempel, eine Norm, ein Ideal, etwas Typisches, in jedem Fall etwas, das sich in aller möglicher Erfahrung gleich bleibt? ${ }^{14}$ So man davon ausgeht, es gehe um Mimesis aller nur vorstellbaren Gegenstände: Ist dieses Allgemeine ein Abstraktum, das sich in allen Modifikationen in der Vorstellung vorfindbarer Gegenstände durchhält, sei dieses Allgemeine nun Ergebnis abstrahierenden Denkens, sei es, dass es in den Gegenständen selbst liege? Wenn aber das Allgemeine ein immer gleiches Abstraktum ist, wie lässt sich dann die Vielfalt der Literatur(en) als Spiegel vielfältiger Erfahrungen begründen? Wird die Darstellung dann nicht irgendwann langweilig? Gibt es überhaupt noch etwas zu sagen? Bedeutet die Bindung der Literatur an die Darstellung eines Allgemeinen nicht den Tod der Literatur?

14 Dies ist die seit der Frühen Neuzeit gängige Auffassung des Allgemeinen als Gegenstand der Literatur; vgl. Brigitte Kappl: "Exemplar vitae - Der Gegenstand von Dichtung bei Aristoteles und seinen Interpreten im Cinquecento«. In: Mimesis - Repräsentation Imagination (Anm. 7), S. 167-180; Ulrike Zeuch: "Aporien in der Literaturtheorie der Frühen Neuzeit. Francesco Robortellos ,In librum Aristotelis de arte poetica explicationes` und die Folgen«. In: Ebd., S. I8I-2I4. 
Dass Aristoteles selbst ein derartiges Abstraktum nicht gemeint hat, sondern den Inbegriff bestimmt unterscheidbarer und erkennbarer Möglichkeiten menschlichen Handelns, dass aber der von ihm in diesem $\mathrm{Zu}$ sammenhang benutzte Allgemeinbegriff in der Rezeption seit der Frühen Neuzeit in diesem Sinne interpretiert worden ist und deshalb zum Stein des Anstoßes werden konnte - dies weiter auszuführen ist hier nicht der Ort. ${ }^{15}$ Tatsache jedenfalls ist, dass die Literatur mit der an sie seit der Frühen Neuzeit gestellten Erwartung, eine immer gleiche Verhaltensnorm darzustellen, Mühe hat; denn zum einen geht sie auf den einzelnen Menschen, zum anderen erkundet Literatur (auch) den Bereich frei imaginierbarer Möglichkeitswelten. ${ }^{16}$

\section{Wieland und die beiden Allgemeinbegriffe}

Was aber macht Wieland aus dem inhomogenen ideengeschichtlichen Erbe? Ich nehme das Ergebnis vorweg: Auf das widersprüchliche Erbe literaturtheoretischer Bestimmungen gibt Wieland eine ebenso inhomogene Antwort.

Gleich zu Beginn des Vorberichts der Geschichte des Agathon destruiert Wieland eine mögliche Lesererwartung: Die Geschichte des Agathon sei nicht authentisch verbürgt. Es bleibe dem Leser überlassen, sich ein eigenes Urteil zu bilden, ob das Erzählte "aus einem alten Griechischen Manuskript " gezogen sei oder nicht. Die Grundlage für ein eigenes Urteil ist jedoch so prekär wie die rhetorisch geschickt verpackte Vergewisserung des Herausgebers selbst, 'nur als Herausgeber zu fungieren. Denn das griechische Manuskript als Vorlage des Erzählten kann ebenso fingiert sein wie das Erzählte selbst.

Mit der Frage der Authentizität ist üblicherweise die Frage nach der Wahrheit des Erzählten, seiner Übereinstimmung mit der Wirklichkeit verknüpft. Hinsichtlich des Wirklichkeitsbegriffs lässt Wieland den Herausgeber eine Unterscheidung zwischen äußerer und innerer Wirklichkeit vornehmen: ,Wirklichkeit könne einerseits äußere Lebensdaten wie Geburt, Heirat, Kinder usw. meinen; das interessiere aber nicht, sei mithin kein Stoff für Literatur. Die reinen Fakten trügen nichts zur Wahrheit bei, die er hier meine. Wahr sei vielmehr die innere Wirklichkeit, die

15 Zur Umdeutung des Begriffs des Allgemeinen als Gegenstand der Literatur seit der Frühen Neuzeit vgl. Zeuch: Das Allgemeine als Gegenstand der Literatur (Anm.2).

16 Wie sehr Wieland der neuzeitlichen Überzeugung des besser Verstehens als der Autor selbst verpflichtet ist, wie sie sich schon im ersten gedruckten Kommentar der Poetik des Aristoteles bei Robortello zeigt, wird an der Zueignungsschrift der Übersetzung der Episteln des Horaz durch Wieland an den Herzog von Sachsen, Carl August, deutlich: Christoph Martin Wieland: „Übersetzung des Horaz«. In: ÜH, S. I2f. 
innere Geschichte Agathons, und zwar die Geschichte seiner charakterlichen Entwicklung, seines Herzens, seiner Leidenschaften, die aufgrund ihrer Individualität und aufgrund der immer anderen Lebensumstände eine ganz spezifische Färbung aufwiesen (GA, S. II).

Eben dieser Agathon, so der Herausgeber weiter, sei der Natur entnommen, sei keine bloße Fiktion, sondern habe ein Vorbild. Dieses Vorbild aber sei nicht historisch belegbar, durch Fakten im Sinne der äußeren Wirklichkeit abgesichert, sondern zeige eine andere Art der Übereinstimmung "mit dem Lauf der Welt" im Sinne der inneren Wirklichkeit (GA, S. II). Diese Übereinstimmung habe zur Folge, dass das, was erzählt werde, selbst wenn alles erfunden sei, dennoch so hätte geschehen können. Sowohl `Natur`wie 'Lauf der Welt`stehen im Vorbericht für etwas Allgemeines, das mit dem absolut Individuellen auf irgendeine Weise zusammenstimme. Ein paar Zeilen weiter aber versichert der Herausgeber, »daß Agathon und die meisten übrigen Personen, welche in seine Geschichte eingeflochten sind, wirkliche Personen sind, dergleichen es von je her viele gegeben hat, und in dieser Stunde noch gibt $[\ldots]$ (... (GA, S. I2).

So soll Agathon einerseits erfunden, andererseits eine historische Person sein, einerseits ist er vorgeblich nicht willkürlich konstruiert, andererseits ist der Lauf der Welt, mit dem Agathons Entwicklung übereinstimmen soll, eine Konstruktion des Herausgebers "nach unserm Plan« (GA, S. I5), einerseits will der Herausgeber einen Menschen darstellen, dessen Charakter heterogene Eigenschaften aufweist und dessen Geschichte eine eigene Dynamik hat, andererseits soll am Ende doch das Modell einer musterhaften Entwicklung zum tugendhaften Mann stehen, einerseits soll Agathon ein absolut unvergleichliches Individuum sein, andererseits ein Mensch, »dergleichen es von jeher viele gegeben hat, und in dieser Stunde noch gibt« (GA, S. I2).

Wie lassen sich diese offensichtlichen Widersprüche deuten? Zwei Antworten darauf liegen nahe: Entweder übernimmt Wieland die aus der Geschichte der Literaturtheorie tradierten Widersprüche (etwas Individuelles, das zugleich allgemein ist, der Inbegriff der Tugend selbst, der zugleich sämtliche, dem Menschen nur mögliche Charaktereigenschaften aufweisen soll, um tatsächlich für alle Menschen repräsentativ sein zu können, die Entwicklung eines Individuums gemäß der für es spezifischen Lebensgeschichte, die zugleich exemplarisch sein soll, die Ablehnung der Fiktion und zugleich die ebenfalls fiktionale, in jedem Fall subjektive Konstruktion einer außersubjektiven Notwendigkeit oder Natur), ohne diese Widersprüche zu bemerken. Oder aber er destruiert durch diese auch für ihn offensichtlichen Widersprüche die Erwartung des Lesers an eine logisch stringente Darlegung der Prinzipien des Herausgebers, wobei dieser 
explizit den Leser mit "gesunden Augen" (GA, S. I4), den "vernünftigen und ehrlichen Leser« (GA, S. I5) anspricht.

Letztere Antwort scheint mir wahrscheinlicher zu sein. Zwar bedarf es einer kritischen, in diesem Sinne vernünftigen Lektüre des Vorberichts, um die Widersprüche überhaupt zu bemerken; Vernunft soll nicht generell suspendiert werden. Aber die Geschichte des Agathon selbst enthält, wie der Herausgeber sagt, so viel unwahrscheinliche Gedanken und Handlungen, dass sie - anders, als der Vorbericht eigentlich glauben machen will -, gerade den Leser fordert, der sich vorbehaltlos auf die Wechselfälle in Agathons Leben einlässt und ihnen zunächst einmal naiv folgt. Die Aufklärung erfolgt dann - durch den Herausgeber wiederum; und auch sie ist, wie schon der Vorbericht zeigt, rationale Plausibilisierung im Nachhinein, die abgetrennt ist vom Fluss der Erzählung, welche die Entwicklung der Gefühle und das mit den Sinnen wahrnehmbare Ambiente beschreibt. Die vom Herausgeber geleistete Aufklärung ist, wie der Vorbericht, in sich widersprüchlich und löst gerade das nicht ein, was sie zu leisten beansprucht: Erhellung von Unbegreiflichem, was sich der Erzähler irgendwann auch eingesteht: "Wie oft sehen wir Personen kommen und wieder abtreten, ohne daß sich begreifen läßt, warum sie kamen, oder warum sie wieder verschwinden? Wie viel wird in beiden dem Zufall überlassen?" (GA, S. 439).

Statt das Unbegreifliche zu erhellen, tritt ein deus ex machina auf, der Gordische Knoten wird zerschnitten statt gelöst, und dann hat "auf die eine oder andere Art das Stück ein Ende« (GA, S. 439). Damit aber wird die eigentliche Aufgabe, das Unbegreifliche zu erhellen, dem Leser überantwortet. ${ }^{17}$

\section{Wieland zwischen den Stühlen}

Wieland ist Horaz' Ars poetica dahingehend verpflichtet, dass er nach wie vor an der Darstellbarkeit der Tugendhaftigkeit, d.i. einer moralischen Haltung selbst festhält; nur ist sie in der Gestalt des Agathon nicht von Anfang an voll ausgebildet präsent, sondern entwickelt sich vor den Augen des Lesers. Wieland zieht damit die naheliegende Konsequenz aus Horaz' Forderung nach sinnlicher Präsenz, nach Augenscheinlichkeit, indem er

17 Frank Baudach: "Die Dichtungsauffassung des jungen Wieland«. In: Dichtungstheorien der deutschen Frühaufklärung. Hg. v. Theodor Verweyen u. Hans-Joachim Kertscher. Tübingen 1995, S. 187-199, hier S. 197, stellt diese Art der >Problemlösung schon in Wielands Schrift Gesicht von einer Welt unschuldiger Menschen von 1755 fest; Wieland zerschlage »den Knoten dort [in dieser Schrift; U.Z.], indem er das Problem praktisch wendet und dem Leser als Aufgabe überantwortet, deren Lösung er durch sein eigenes Handeln anstreben muß«. 
das Werden eines Tugendhaften darstellt und die Statik, die Horaz' Allgemeinbegriff eignet, zugunsten einer dynamisierten Variante transformiert, die zugleich einer Begriffsbildung, in diesem Fall dessen, was der Mensch ist, der "Kenntnis des Menschen" (GA, S. 497), mit Hilfe der Empirie Rechnung zu tragen beansprucht.

Die Priorität der Empirie, die Wieland in der Vorrede dezidiert gegenüber apriorischen ideellen Vorgaben vertritt, ist im Kontext der Platon-Rezeption seit der Frühen Neuzeit und der kritischen Auseinandersetzung mit dem Platonismus beziehungsweise mit dem, was man für platonisch hält, zu sehen. Indem in den Humanitätsdiskursen der Aufklärung davon ausgegangen wird, dass es so etwas Allgemeines wie eine Menschheit und einen Zweck des Menschen überhaupt gibt, nämlich als Mensch nach dem für ihn als Menschen Besten zu streben, dass dieser Zweck für den Menschen erkennbar und es die Aufgabe des Menschen sei, diesen erkannten Zweck in sich zu verwirklichen, für sich individuell auszubilden, übernimmt er eine Position des seit der Frühen Neuzeit von Marsilio Ficino, Giordano Bruno und anderen entwickelten Platonismus. ${ }^{18}$

Die spezielle Neudeutung des frühneuzeitlichen Platonismus ist in der Annahme begründet, dass die Ideen der Materie, den einzelnen Phänomenen, immanent und in ihnen auch erkennbar seien. Indem davon ausgegangen wird, dass die Ideen als solche in der Materie offenbar würden, wird dem Vorrang der Wahrnehmung, der Erfahrung und Empirie vor der Abstraktion im Erkenntnisprozess Rechnung getragen, wie er in den einzelnen Wissenschaften als methodischer Zugang seit der Frühen Neuzeit propagiert wird. ${ }^{19}$

Ich kann an dieser Stelle nicht in extenso auf die erkenntnistheoretischen Probleme eingehen, die mit der Annahme einer Immanenz der Ideen in der Materie einhergehen. Hervorheben möchte ich jedoch, dass genau dies ein zentraler Ansatzpunkt für die Kritik am Humanitätsbegriff der Aufklärung in der Nachfolgezeit ist: Wenn das, was der Mensch ist bzw. sein soll, in der Materie selbst, in den einzelnen Verwirklichungen des Menschseins ablesbar sein soll, in der Erfahrung aber einander widersprechende, ja miteinander unvereinbare Verwirklichungen angetroffen werden, dann liegt der Schluss nahe, dass es diese Idee als normative und teleologische Vorgabe selbst nicht gibt.

18 Ulrike Zeuch: „Herders Begriff der Humanität: aufgeklärt und aufklärend über seine Prämissen? Zur Bestimmung des höchsten Zwecks des Menschen in den >Ideen und in der ’Oratio von Giovanni Pico delle Mirandola«. In: Vom Selbstdenken. Aufklärung und Aufklärungskritik in Herders ,Ideen zur Philosophie der Geschichte der Menschheitr. Hg. v. Regine Otto u. John H. Zammito, Heidelberg 200I, S. 187-198.

19 Zu Ficinos Ideenbegriff vgl. Ulrike Zeuch: Umkehr der Sinneshierarchie. Herder und die Aufwertung des Tastsinns seit der frühen Neuzeit. Tübingen 2000, S. I83ff. 
Nun ließe sich einwenden, dass sich ein Abweichen von der Norm, von dem, was das Menschsein ausmachen soll, nur erkennen lässt, wenn es zumindest einen vagen Vorbegriff a priori gibt, wie bei Kant etwa die systematischen, empirisch nicht gegebenen »drei regulativen Ideen der spekulativen Vernunft«: Seele, Welt und Gott. ${ }^{20}$ Ansonsten hätte man kein Beurteilungskriterium, anhand dessen sich die Erscheinungen bewerten ließen. Eben dieses Dilemma ist der Erkenntnistheorie der Aufklärung eigen: Eigentlich kommt der Mensch nur über die Wahrnehmung und die in der Vorstellung erinnerten Wahrnehmungsmomente, die unter einen Begriff subsumiert werden, zu einem inhaltlich angereicherten Begriff dessen, was der Mensch ist bzw. sein soll. Zugleich aber muss er auf irgendeine Weise schon wissen, was er sucht, sonst könnte er seine Erfahrungen nicht ordnen, Dazugehörendes sammeln, Nichtdazugehörendes als Akzidenzien absondern, Widersprechendes aussondern usf.

Wieland versucht, dieses Dilemma zu lösen, indem er davon ausgeht, es habe, zumindest bedingt, in der Geschichte der Menschheit schon einmal eine solche in der Erfahrung ablesbare Verwirklichung der Idee der Menschheit gegeben: in Agathon. Zwar mache dieser »kein Modell eines vollkommen tugendhaften Mannes", aber es gebe auch keinen, der "unter allen nach dem natürlichen Lauf Gebornen, in ähnlichen Umständen, und alles zusammen genommen, tugendhafter gewesen wäre, als Agathon« (GA, S. I3).

Damit gerät Wieland aber in ein zweites Dilemma: die Gleichwertigkeit der Verwirklichungen des Menschseins in ihrer Vielgestaltigkeit mit der Vorgabe einer real gewordenen Norm, einem leibhaften Maßstab zu vereinbaren.

In seinen antiplatonischen ${ }^{21}$ Briefen an Eurybates (AZ, S. $748 \mathrm{ff}$.) legt Aristipp eben dieses Dilemma in der Auseinandersetzung mit Platons Politeia dar.

Der Mensch läßt sich nicht, wie eine regelmäßige geometrische Figur, in etliche scharf gezogene gerade Linien einschließen; und es sind vielleicht noch Jahrtausende einer anhaltenden, eben so unbefangenen als scharfsichtigen Beobachtung unsrer Natur vonnöten, bevor es möglich sein wird, nur die Grundlinien zu einem echten Modell der besten gesellschaftlichen Verfassung für die

20 Immanuel Kant: »Kritik der reinen Vernunft (I. Aufl. I78I)«. In: Kants Werke. AkademieTextausgabe. Unveränderter photomechanischer Abdruck des Textes der von der Preußischen Akademie der Wissenschaften 1902 begonnenen Ausgabe von Kants gesammelten Schriften, Bd. 4. Hg. v. der Preußischen Akademie der Wissenschaften, Berlin I968, A 682 / B 710 im Kapitel "Von der Endabsicht der natürlichen Dialektik«; allgemeiner, was Kant unter einer Idee versteht, vgl. B $384 /$ A $327 \mathrm{ff}$. "Von den transzendentalen Ideen«; dort werden die drei Bereiche Psychologie, Kosmologie, Theologie auch noch einmal angeführt.

21 Zur Kritik Wielands an Platon vgl. Klaus Manger: Klassizismus und Aufklärung: Das Beispiel des späten Wieland. Frankfurt/M. 1991, S. I44f., I79ff. 
wirklichen Menschen zu zeichnen; und selbst dieses Modell würde für jedes besondere Volk, durch dessen eigene Lage und die Verschiedenheit der Zeit- und Ortumstände, auch verschiedentlich bestimmt und abgeändert werden müssen. Aber auf alles dies nimmt ein Platon keine Rücksicht [...] (AZ, S. 755).

Wieland bleibt auch dahingehend Horaz verpflichtet, als an einem Individuum exemplarisch etwas Allgemeines ablesbar sein soll, denn "die Geschichte Agathons", so heißt es an einer Stelle, sei »die Geschichte aller Menschen" (GA, S. 503). Mit der Dynamisierung aber entgleitet Wieland das Horaz'sche Allgemeine als konstant gleichbleibendes Ideal; mit der Aufgabe des Wahrscheinlichkeitspostulats als Wahrheitskriterium zugunsten des Unbegreiflichen entfällt Horaz' Legitimation der Literatur als Darstellung von Wahrem.

In der Einleitung zum ersten Brief des Horaz an C. Cilnius Maecenas zeichnet Wieland Maecenas als einen mittleren Charakter, weder besonders vorbildhaft noch reduzierbar auf eine hervorragende Eigenschaft. In der Mischung der durchaus heterogenen Eigenschaften zeigt sich für Wieland etwas Allgemeines in dem Sinne, dass man sich den Maecenas der Antike nicht anders denken müsse, als wie "Personen von seinen Umständen auch in unseren Zeiten zu sein pflegen «. ${ }^{22}$ Maecenas als Substrat, von dem Verschiedenes prädiziert wird, ist Horaz verpflichtet. Indem aber die Heterogenität, ja Disparatheit eines komplexen Charakters betont wird, entgleitet Wieland das Horaz'sche Allgemeine als widerspruchsloses Eines.

Im Aristipp zieht Wieland daraus folgende Konsequenz: Während Horaz, wie Wieland ihn im Kommentar zum I7. Brief des I. Buches der Horaz'schen Briefe auslegt, Aristipp als »Muster und Ideal eines >Philosophen ‘ am Hofe darstellt« (ÜH, S. 262), ist der Wieland'sche Aristipp im gleichnamigen Roman Aristipp und einige seiner Zeitgenossen keineswegs als reines Ideal oder Muster konzipiert und dargestellt. Im Vorwort zum I. Brief der I. Buches der Briefe des Horaz enthält Wieland sich sogar explizit eines Urteils: »Ich untersuche hier nicht, ob diese ziemlich sunpoetische Art zu philosophieren die beste sei; ich sage nur: dies war Aristipps Philosophie (ÜH, S. 6I)."Um seine Enthaltung eines Urteils, welches Aristipp eine Monopolstellung zuweisen würde, glaubwürdig zu machen, stellt Wieland Aristipps moralphilosophische Haltung in den Kontext des von Wieland konstruierten bzw. rekonstruierten und breit angelegten Diskurses über die Tauglichkeit verschiedener, zeitgenössischer Philosophien hinsichtlich ihrer lebenspraktischen ethischen Relevanz.

Gleichwohl lässt Wieland durch die Art, wie er den Diskurs führen lässt, keinen Zweifel, wem seine Sympathie gilt: Aristipp, und zwar wegen dessen Präferenz der Lebenspraxis, seines aus Erfahrung abgeleiteten

22 Christoph Martin Wieland: „Briefe des Horaz«. In: ÜH, S. 34. 
Wissens, seiner Offenheit und Dialogbereitschaft - mit diesen Charaktereigenschaften stattet ihn Wieland zumindest in der Romanfiktion aus. Die Heterogenität, Disparatheit bzw. Widersprüchlichkeit der Haltungen ist dabei - auch dies eine Konsequenz aus dem Dilemma mit dem Romankonzept des Agathon - konsequent von der Handlungsebene auf die Diskursebene verlagert, die kritische Stimmen pro und contra Aristipp vereinen. Letztlich gilt für den Wahrheitsanspruch der Literatur als Medium eines derartigen Diskurses, was Wieland Diogenes in den Mund legt: "Jeder hat für seine eigene Person Recht; aber so bald sie miteinander hadern, und sich um den ausschließlichen Besitz der Wahrheit, wie Hunde um einen fetten Knochen, herumbeißen, dann haben sie alle Unrecht [...].« (AZ, S. 865f.) Angesichts der impliziten Distanzierung von Horaz' Allgemeinbegriff der Literatur würde naheliegen, dass Wieland nach Alternativen theoretischer Begründung von Literatur, etwa in Auseinandersetzung mit dem Allgemeinbegriff des Aristoteles, sucht. Das hat er nicht getan. Im dritten der Briefe an einen jungen Dichter ${ }^{23}$ von 1784 findet sich der mögliche Grund dafür. Literatur soll, schreibt Wieland in diesem Brief, den Blick in "die innersten Falten des Herzens" offenlegen (SW XIV.6, S. 272) und lebendig, reich an Bildern und Gedanken sowie mit innerer Anteilnahme den Menschen darstellen (SW XIV.6, S. 273). Die »Stärke aller Seelenkräfte, an innigem Gefühl der Natur" (SW XIV.6, S. 274) als Voraussetzung für Literatur in Wielands Sinne betont er mehrfach. Nur: Dieses Gefühl lässt sich weder erwerben noch durch Regeln erlernen noch nachahmen (SW XIV.6, S. 276). Und: Literatur, die diesem Maßstab genügt, wie die Dramen Shakespeares, so Wieland, sind zwar selbst "Abdrücke der Natur" (SW XIV.6, S. 276); es macht aber keinen Sinn, sie I : I nachzubilden. Sie können als »Modelle« (SW XIV.6, S. 277) betrachtet werden; sie sind aber nicht identisch übertragbar.

Da Wieland Aristoteles in Gottscheds Nachfolge als Regelpoeten begreift, ${ }^{24}$ ist die Poetik des Aristoteles für die Literatur, die Wieland favorisiert, nicht der geeignete Leitfaden. Stattdessen versucht er, Horaz' Allgemeines so zu deuten, d.h. eigentlich - wie schon Francesco Robortello

23 Christoph Martin Wieland: "Briefe an einen jungen Dichter». In: Ders.: Sämmtliche Werke. Hg. v. d. "Hamburger Stiftung zur Förderung von Wissenschaft und Kultur» in Zusammenarbeit m. d. "Wieland-Archiv«, Biberach/Riß u. Hans Radspieler. Hamburg u.a.: 1984. 36 Bde. u. 6 Supplementbände [Faksimiledruck der Sämmtlichen Werke, Leipzig 1794-I8II], erschienen in I4 Bänden. (Hier im Text zitiert mit der Sigle SW, römischer Zahl für Band, arabischer Zahl für Originalband sowie Seitenzahl.) Bd. I4, Supplement 6, S. 267-296.

24 Ulrike Zeuch: „Dichtungstheorie der Frühaufklärung«. In: Aufklärung I7 (2005), S. II7-I40; Dies.: »Bewußtseinsphilosophische Prämissen der Literaturtheorie vor I80o. Am Beispiel von Gottscheds , Versuch einer Critischen Dichtkunst und deren Folgen«. In Zeitschrift für Ästhetik und allgemeine Kunstwissenschaft 46/I (200I), S. 53-75. 
in dem gedruckten Kommentar zur aristotelischen Poetik der Frühen Neuzeit - umzudeuten, zu transformieren (besser zu verstehen als der Autor selbst), dass es zur Anforderung der eigenen Zeit passt, die weder als homogen noch kontinuierlich noch einfach wahrgenommen wird. Oder in den Worten von Wieland selbst: "Nicht darin daß wir schlechte Muster genommen, sondern daß wir den guten größtenteils auf einem verkehrten Wege und auf eine verkehrte Art nachgeahmt haben, liegt das Übel [...].« (SW XIV.6, S. 278) Diese produktive Transformation hat Wieland Horaz' Ars poetica zuteil werden lassen, Aristoteles' Poetik nicht. Aber auch literarisch entspricht Wieland nicht dem Aristotelischen Allgemeinen, wenn er im Aristipp als Gegenstand der Darstellung definiert, was eine Person ihrem Wesen nach ausmacht bzw. diese wesentlich bestimmt, so dass diese Wesensart durchgängig alle ihre Handlungen bestimmt, wie Aristipp über die Hetäre Lais ausführt.

Die Natur schickt uns [...] mit lauter unbestimmten Anlagen in die Welt [...] Indessen würde doch, glaube ich, ein Gott, der das ganze, uns unsichtbare Gewebe der innern Anlagen eines Menschen zu durchschauen vermöchte, das, wozu ihn diese Anlagen vor allem andern bestimmen, unfehlbar entdecken; denn in der Natur gibt es nichts wirklich unbestimmtes. Je lebendiger also das Selbstgefühl bei einer Person ist, desto mehr ist zu vermuten, daß sie, wenn die äußern Umstände ihr völlig Freiheit lassen, sich selbst für diejenige Lebensweise bestimmen werde, zu welcher sie durch ihre ganze Naturanlage vor allem andern geschickt gemacht ist (AZ, S. 62I).

Lais habe sich zugetraut, "den außerordentlichen Karakter, worin sie in der Welt auftreten wollte, immer behaupten zu können" (AZ, S. 622). Es ist eben dieser Begriff des individuellen Allgemeinen, der dank des durch den stoischen Allgemeinbegriff bestimmten Horaz und des Nominalismus des Spätmittelalters an die Frühe Neuzeit vermittelt wird und trotz grundsätzlicher Probleme, wie dargelegt, unangefochten gültig bleibt, nurmehr erfassbar durch das Gefühl bzw. das Selbstgefühl.

Insofern stellt sich - dies mein Resümee - Wielands Position innerhalb des anfangs skizzierten Problemfeldes als wenig homogen dar; zwar folgt er Horaz nominell, unterschwellig aber transformiert er ihn. Diese Transformation geht aber nicht so weit, nach einer grundsätzlichen Alternative zu forschen. Wielands Unentschiedenheit lässt sich durchaus als Signum eines Übergangs, desjenigen von der Frühmoderne zur Moderne, begreifen, als Versuch, die Autonomie des Lesers ernst zu nehmen. Deshalb wählt er eine Erzählform, das Gespräch in Briefen, die sich durch Polyvalenz, Multiperspektivik und Intersubjektivität auszeichnet, diese legitimiert und die historisch zurückliegende mit zeitgenössischen Sinnund Deutungsangeboten kontrastiert wie verbindet. Es gibt nicht die eine verbindliche Konstruktions- und Lesart von Wirklichkeit und Fiktion; es gibt nicht den monologischen Sprecher und nicht den privilegierten 
Adressaten oder Leser. Das Gespräch ist ergebnisoffen, mithin unendlich fortsetzbar. Das ist Wielands komplexe Antwort auf die komplexe Frage nach der Wahrheit der Literatur. 